\title{
Poor and pi-poor Abelian groups
}

\author{
Rafail Alizade ${ }^{a}$ and Engln Büyükaşık

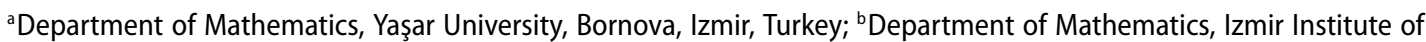 \\ Technology, Urla, Izmir, Turkey
}

\begin{abstract}
In this paper, poor abelian groups are characterized. It is proved that an abelian group is poor if and only if its torsion part contains a direct summand isomorphic to $\oplus_{p \in P} \mathbb{Z}_{p}$, where $P$ is the set of prime integers. We also prove that pi-poor abelian groups exist. Namely, it is proved that the direct sum of $U^{(\mathbb{N})}$, where $U$ ranges over all nonisomorphic uniform abelian groups, is pipoor. Moreover, for a pi-poor abelian group $M$, it is shown that $M$ can not be torsion, and each $p$-primary component of $M$ is unbounded. Finally, we show that there are pi-poor groups which are not poor, and vise versa.
\end{abstract}

\section{ARTICLE HISTORY}

Received 6 May 2015

Revised 3 November 2015

Communicated by A. Facchini

\section{KEYWORDS}

Injective module; pi-poor abelian groups; poor abelian groups; pure-injective module

\section{MATHEMATICS} SUBJECT CLASSIFICATION 13C05; 13C11; 13C99; 20E34; 20 E99

\section{Introduction}

Let $R$ be a ring with an identity element and $\operatorname{Mod}-R$ be the category of right $R$-modules. Recall that a right $R$-module $M$ is said to be an $N$-injective (or injective relative to $N$ ) if for every submodule $K$ of $N$ and every morphism $f: K \rightarrow M$ there exists a morphism $\bar{f}: N \rightarrow M$ such that $\left.\bar{f}\right|_{K}=f$. For a module $M$, as in [2], the injectivity domain of $M$ is defined to be the collection of modules $N$ such that $M$ is an $N$-injective, that is, $\mathfrak{I n}^{-1}(M)=\{N \in \operatorname{Mod}-R \mid M$ is $N$-injective $\}$. Clearly, for any right $R$-module $M$, semisimple modules in $M o d-R$ are contained in $\mathfrak{I n}^{-1}(M)$, and $M$ is an injective if and only if $\mathfrak{I n}^{-1}(M)=\operatorname{Mod}-R$. Following [1], $M$ is called poor if for every right $R$-module $N, M$ is an $N$-injective only if $N$ is semisimple, i.e., $\mathfrak{I n}^{-1}(M)$ is exactly the class of all semisimple right $R$-modules. Poor modules exist over arbitrary rings [3, Proposition 1]. Although poor modules exist over arbitrary rings, their structure is not known over certain rings including also the ring of integers.

A right $R$-module $N$ is pure-split if every pure submodule of $N$ is a direct summand. Let $K$ and $N$ be right $R$-modules. $K$ is an $N$-pure-injective if for each pure submodule $L$ of $N$ every homomorphism $f: L \rightarrow K$ can be extended to a homomorphism $g: N \rightarrow K$. Following [7], a right $R$-module $M$ is called pure-injectively poor (or simply pi-poor) if whenever $M$ is an $N$-pure-injective, then $N$ is pure-split. It is not known whether pi-poor modules exist over arbitrary rings. In particular, in [7], some classes of abelian groups that are not pi-poor are given but the authors point out that they do not know whether a pi-poor abelian group exists.

The purpose of this paper is to give a characterization of poor abelian groups and also to prove that pi-poor abelian groups exist.

Namely, in Section 3, we prove that an abelian group $G$ is poor if and only if the torsion part of $G$ contains a direct summand isomorphic to $\oplus_{p \in P} \mathbb{Z}_{p}$, where $P$ is the set of prime integers (Theorem 3.1).

Section 4 is devoted to the proof of the existence of pi-poor abelian groups. Let $\left\{A_{\gamma} \mid \gamma \in \Gamma\right\}$ be a complete set of representatives of isomorphism classes of reduced uniform groups. We prove that the 
group $M=\bigoplus_{\gamma \in \Gamma} A_{\gamma}^{(\mathbb{N})}$ is pi-poor (Theorem 4.1). In addition, it is proved that if $G$ is a pi-poor abelian group, then $G$ is not torsion, and the $p$-primary component $T_{p}(G)$ of $G$ is unbounded for each prime $p$.

\section{Definitions and preliminaries}

We recall some definitions and results which will be useful in the sequel. For more details, we refer the reader to [5]. By group, we will mean an abelian group throughout the paper. Let $p \in P$ be a prime integer. A group $G$ is called $p$-group if every nonzero element of $G$ has order $p^{n}$ for some $n \in \mathbb{Z}^{+}$. For a group $G, T(G)$ denotes the torsion submodule of $G$. The set $T_{p}(G)=\left\{a \in G \mid p^{k} a=0\right.$ for some $k \in$ $\left.\mathbb{Z}^{+}\right\}$is a subgroup of $G$, which is called the p-primary component of $G$. For every torsion group $G$, we have $G=\oplus_{p \in P} T_{p}(G)$. A subgroup $A$ of a group $B$ is pure in $B$ if $n A=A \cap n B$ for each integer $n$. A monomorphism (resp. epimorphism) $\alpha: A \rightarrow B$ of abelian groups is called pure if $\alpha(A)(\operatorname{resp}$. $\operatorname{Ker}(\alpha))$ is pure in $B$. For any group $G$, the subgroups $T(G)$ and $T_{p}(G)$ are pure in $G$. A group $G$ is said to be bounded if $n G=0$, for some nonzero integer $n$. Bounded groups are direct sum of cyclic groups [5, Theorem 17.2]. A group $G$ is called a divisible group if $n G=G$ for each positive integer $n$. A group $G$ is called a reduced group if $G$ has no proper divisible subgroup. Note that, since $\mathbb{Z}$ is Noetherian, every group $G$ contains a largest divisible subgroup. Therefore, $G$ can be written as $G=N \oplus D$, where $N$ is reduced and $D$ is divisible subgroup of $G$.

Definition 2.1 (see [5]). Let $p \in P$. A subgroup $B$ of a group $A$ is called a $p$-basic subgroup of $B$ if it satisfies the following three conditions:

(i) $B$ is a direct sum of cyclic $p$-groups and infinite cyclic groups;

(ii) $B$ is $p$-pure in $A$;

(iii) $\quad A / B$ is $p$-divisible, i.e., $p(A / B)=A / B$.

\section{Lemma 2.2.}

(a) [5, Theorem 32.3] Every group $G$ contains a $p$-basic subgroup for each $p \in P$.

(b) [5, Theorem 27.5] If $H$ is a pure and bounded subgroup of a group $G$, then $H$ is a direct summand of $G$.

For $q \neq p q$-basic subgroups of $p$-groups are 0 , so only $p$-basic subgroups of $p$-groups may be nontrivial. Therefore, they are usually called simply basic subgroups. Clearly, basic subgroups of $p$ groups are pure. Subgroups of the group of the rational integers $\mathbb{Q}$ are called rational groups. Let $A$ be a uniform group. Then, it is easy to see that either $A$ is isomorphic to a rational group or $A \cong \mathbb{Z}_{p^{n}}$ for some $p \in P$ and $n \in \mathbb{Z}^{+}$. For a torsion-free group $G$, we shall denote the (torsion-free) rank (=uniform dimension) of $G$ by $r_{0}(G)$ [5]. By [5, page 86, Example 3], $r_{0}(G)=r_{0}(H)+r_{0}(G / H)$ for each subgroup $H$ of $G$. A torsion-free group $G$ is said to be completely decomposable if $G=\bigoplus_{i \in I} K_{i}$, where $I$ is an index set and each $K_{i}$ is isomorphic to a rational group, i.e., $r_{0}\left(K_{i}\right)=1$ for each $i \in I$.

\section{Poor Abelian groups}

In this section, we give a characterization of poor groups. The authors prove that the group $\oplus_{p \in P} \mathbb{Z}_{p}$ is poor [1]. The following result shows that this group is crucial in investigation of poor groups.

Theorem 3.1. A group is poor if and only if its torsion part has a direct summand isomorphic to $\oplus_{p \in P} \mathbb{Z}_{p}$.

Proof. To prove the necessity, let $G$ be a poor group and let $p$ be any prime. If $T_{p}(G)=0$, then $G$ is an $N$-injective for every $p$-group $N$, therefore $T_{p}(G) \neq 0$. If every element of order $p$ of $G$ is divisible by $p$, then $G$ is $\mathbb{Z}_{p^{2}}$-injective since $\mathbb{Z}_{p^{2}}$ has only one nontrivial subgroup: $p \mathbb{Z}_{p^{2}}$. So there is at least one element $a_{p}$ with $\left|a_{p}\right|=p$, that is, not divisible by $p$. Then the cyclic group $<a_{p}>$ is a $p$-pure subgroup of $T_{p}(G)$, therefore a pure subgroup of $T_{p}(G)$. Since bounded pure subgroups are direct summands, $<a_{p}>$ is a 
direct summand of $T_{p}(G)$. Hence $\oplus_{p \in P}<a_{p}>$ is a direct summand of $\oplus_{p \in P} T_{p}(G)=T(G)$. Clearly, $\oplus_{p \in P}<a_{p}>\cong \oplus_{p \in P} \mathbb{Z}_{p}$.

Conversely, suppose that $T(G)$ contains a direct summand isomorphic to $\oplus \mathbb{Z}_{p}$. Let $V$ be a direct summand of $T(G)$ such that $V \cong \mathbb{Z}_{p}$. Then, $V$ is pure in $G$ because $T(G)$ is pure in $G$. So $V$ is a direct summand in $G$ by [5, Theorem 27.5]. This implies, for each prime $p, G$ contains a direct summand isomorphic to $\mathbb{Z}_{p}$. Now, suppose $G$ is an $N$-injective for some group $N$. Then $\mathbb{Z}_{p}$ is an $N$-injective for each prime $p$. Suppose that $N$ is not semisimple (not elementary in terminology of [5]). Then, there is an element $a$ of infinite order or with $o(a)=p^{n}$, where $p$ is a prime and $n>1$. In first case, $\langle a\rangle=\mathbb{Z}$

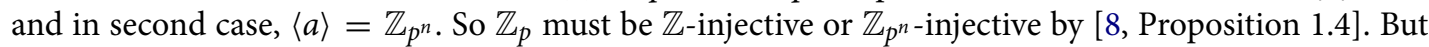
the homomorphism $f: p \mathbb{Z} \rightarrow \mathbb{Z}_{p}$ with $f(p)=1$ cannot be extended to $g: \mathbb{Z} \rightarrow \mathbb{Z}_{p}$ since otherwise $1=f(p)=g(p)=p g(1)=0$ and $\mathbb{Z}_{p}$ is isomorphic to the subgroup $\left\langle p^{n-1}\right\rangle$ of $\mathbb{Z}_{p^{n}}$, which is not a direct summand of $\mathbb{Z}_{p^{n}}$. So in both cases we get a contradiction, that is, $N$ is semisimple.

The following is a consequence of Theorem 3.1.

Corollary 3.2. For a group $G$, the following are equivalent.

(1) $G$ is poor.

(2) The reduced part of $G$ is poor.

(3) $T(G)$ is poor.

(4) For each prime $p$, G has a direct summand isomorphic to $\mathbb{Z}_{p}$.

\section{Pi-poor Abelian groups}

The authors investigate the notion of pi-poor module and study properties of these modules over various rings [7]. In particular, they give some classes of groups that are not pi-poor and point out that they do not know whether a pi-poor group exists or not. In this section, we shall prove that pi-poor groups exist.

Theorem 4.1. Let $\left\{A_{\gamma} \mid \gamma \in \Gamma\right\}$ be a complete set of representatives of isomorphism classes of uniform groups. Then the group

$$
M=\bigoplus_{\gamma \in \Gamma} A_{\gamma}^{(\mathbb{N})}
$$

is pi-poor.

Before proving the theorem, we will first give some lemmas. Throughout this section, $M$ denotes the group given in Theorem 4.1 .

The following result is well known. We include it for completeness.

Lemma 4.2. Let $R$ be a ring and $L, N$ be right $R$-modules. Let $K$ be a pure submodule of $N$. If $L$ is an $N$-pure-injective, then $L$ is both $K$-pure-injective and $N / K$-pure-injective.

Proof. Let $A$ be a pure submodule of $K$ and $f: A \rightarrow L$ be a homomorphism. Then $A$ is pure in $N$, and so $f$ extends to a map $g: N \rightarrow L$. Clearly, $\left.g\right|_{K}: K \rightarrow L$ is an extension of $f$ to $K$. Hence $L$ is $K$-pureinjective. Now, let $X / K$ be a pure submodule of $N / K$ and $f: X / K \rightarrow L$ be a homomorphism. Since $K$ is pure in $N$ and $X / K$ is pure in $N / K, X$ is pure in $N$. Therefore, there is a homomorphism $g: N \rightarrow L$ such that $f \pi^{\prime}=g i$, where $i: X \rightarrow N$ is the inclusion and $\pi^{\prime}: X \rightarrow X / K$ is the usual epimorphism. Since $g(K)=0, \operatorname{Ker}(\pi) \subseteq \operatorname{Ker}(g)$, where $\pi: N \rightarrow N / K$ is the usual epimorphism. Therefore, there is a homomorphism $h: N / K \rightarrow L$ such that $h \pi=g$. Then for each $x \in X, h(x+K)=h(\pi(x))=g(x)=$ $\left(f \pi^{\prime}\right)(x)=f(x+K)$. That is, $h$ extends $f$. Hence, $L$ is an $N / K$-pure-injective. 
Lemma 4.3. Let $G$ be a reduced torsion group. The following are equivalent.

(1) $M$ is G-pure-injective.

(2) $T_{p}(G)$ is bounded for each $p \in P$.

(3) $G$ is pure-split.

Proof.

(1) $\Rightarrow$ (2) Write $G=\bigoplus_{p \in P} T_{p}(G)$. Let $B_{p}(G)$ be a basic subgroup of $T_{p}(G)$. Then $B_{p}(G)$ is pure in $T_{p}(G)$, and so in $G$ and $T_{p}(G) / B_{p}(G)$ is divisible. We claim that $B_{p}(G)$ is bounded. Suppose the contrary that $B_{p}(G)$ is not bounded. Then for every positive integer $n, B_{p}(G)$ contains an element of order $p^{n}$. In this case, since $B_{p}(G)$ is a direct sum of cyclic $p$-groups, there is an epimorphism

$$
B_{p}(G) \stackrel{g}{\rightarrow} \mathbb{Z}_{p} \infty \rightarrow 0,
$$

where the restrictions of $g$ to the cyclic summands of $B_{p}(G)$ are monic. It can be proved as in [5, Lemma 30.1] that $g$ is a pure epimorphism, i.e., $K=\operatorname{Ker}(g)$ is a pure submodule of $B_{p}(G)$. Now, $K$ is pure in $B_{p}(G)$ and is a direct sum of cyclic $p$-groups. Since $M$ contains a direct summand isomorphic to $K$, and $B_{p}(G)$ is a pure subgroup of $G, K$ is $B_{p}(G)$-pure-injective. Therefore $B_{p}(G) \cong K \oplus \mathbb{Z}_{p^{\infty}}$. This contradicts with the fact that $B_{p}(G)$ is reduced. Hence $B_{p}(G)$ is bounded, and so $B_{p}(G)$ is a direct summand of $G$. The fact that $G$ is reduced and $T_{p}(G) / B_{p}(G)$ divisible implies that $B_{p}(G)=T_{p}(G)$.

(2) $\Rightarrow$ (3) Let $H$ be a pure subgroup of $G$. Since $G=\oplus_{p \in P} T_{p}(G)$ and $H=\oplus_{p \in P} T_{p}(H), T_{p}(H)$ is a pure subgroup of $T_{p}(G)$. Then, $T_{p}(H)$ is a direct summand of $T_{p}(G)$ by [5, Theorem 27.5]. Let $T_{p}(G)=T_{p}(H) \oplus N_{p}$, where $N_{p} \leq G$. Then $G=\oplus_{p \in P}\left[T_{p}(H) \oplus N_{p}\right]=\left(\oplus_{p \in P} T_{p}(H)\right) \oplus\left(\oplus_{p \in P} N_{p}\right)=$ $H \oplus\left(\oplus_{p \in P} N_{p}\right)$. Hence $G$ is pure-split.

(3) $\Rightarrow$ (1) Clear by the definition.

Remark 4.4. Pure-split groups are completely characterized in [4]. The implications (2) $\Leftrightarrow(3)$ in Lemma 4.3 also can be found in [4].

Lemma 4.5. Let B be a p-group. Suppose that $M$ is B-pure-injective. Then B is pure-split.

Proof. Let $D$ be the divisible subgroup of $B$ and $A$ be a pure subgroup of $B$. Then $B=C \oplus D$ for some reduced group $C$. Let $D_{A}$ be the divisible subgroup of $A$. Then $D_{A} \leq D$ and $D=D_{1} \oplus D_{A}$ for some $D_{1} \leq D$. So $B=C \oplus D_{1} \oplus D_{A}=E \oplus D_{A}$, where $E=C \oplus D_{1}$. By modular law, $A=(E \cap A) \oplus D_{A}$. Then $L=E \cap A$ is a pure submodule of $B$. Hence, $M$ is $L$-pure-injective, and $L \cong A / D_{A}$ is reduced. Therefore, $L$ is bounded by Lemma 4.3. Since $L$ is pure in $B, L$ is also pure in $E$. Then, $E=K \oplus L$ for some $K \leq E$ by [5, Theorem 27.5]. Then $B=E \oplus D_{A}=K \oplus L \oplus D_{A}=K \oplus A$. So $A$ is a direct summand in $B$. Hence $B$ is pure-split.

Lemma 4.6. If $N$ is a reduced torsion-free group such that $M$ is an $N$-pure-injective then $N$ is pure-split. Moreover, $N$ is completely decomposable with finite rank.

Proof. Take any $0 \neq a_{1} \in N$ and let $G_{1}=\left\{x \in N \mid m x \in\left\langle a_{1}\right\rangle\right.$ for some $\left.0 \neq m \in \mathbb{Z}\right\}$ (that is, $G_{1}$ is the subgroup purely generated by $a_{1}$ ). Clearly, $G_{1}$ is a pure subgroup of $N$ and isomorphic to a rational group, so $M$ has a direct summand isomorphic to $G_{1}$. Therefore, $G_{1}$ is a direct summand of $N$, that is, $N=G_{1} \oplus N_{1}$ for some $N_{1} \leq N$. If $N_{1} \neq 0$, we can find in similar way a pure subgroup $G_{2}$ of $N_{1}$ purely generated by an element $a_{2}$. Clearly, $M$ is an $N_{1}$-pure-injective, so $N_{1}=G_{2} \oplus N_{2}$. The same can be done for $N_{2}$ if $N_{2} \neq 0$ and so on. If this process continues infinitely, then $N$ contains a subgroup $\oplus_{i=1}^{\infty} G_{i}$ which is pure as a direct limit of pure subgroups. Therefore, $M$ is $\oplus_{i=1}^{\infty} G_{i}$-pure-injective. For each $a_{i}$, $i=1,2, \ldots$, there is a homomorphism $f_{i}:\left\langle a_{i}\right\rangle \rightarrow \mathbb{Q}$ with $f\left(a_{i}\right)=\frac{1}{i}$. Since $\mathbb{Q}$ is an injective, there is 
a homomorphism $f: \oplus_{i=1}^{\infty} G_{i} \rightarrow \mathbb{Q}$ with $f\left(a_{i}\right)=f_{i}\left(a_{i}\right)=\frac{1}{i}$. Clearly, $f$ is an epimorphism. Since $\mathbb{Q}$ is torsion-free, $K=\operatorname{Ker}(f)$ is a pure subgroup of $\oplus_{i=1}^{\infty} G_{i}$. Let $\Gamma$ be the set of all completely decomposable pure subgroups of $K$ and $R$ be the set of all subgroups of $K$ of rank 1. Define order $\preceq$ on $\Gamma$ as follows: $\oplus_{S \in I} S \preceq \oplus_{S \in J} S$ if $I \subseteq J \subseteq R$. If $P$ is any chain in $\Gamma$, then $\cup_{X \in P} X$ is clearly a completely decomposable and pure subgroup of $K$, since the direct limit of pure subgroups is pure. So by Zorn's Lemma, there is a maximal element $B=\oplus_{S \in T} S$ in $\Gamma$. Since $K$ is countable $T$ is also countable, so $B$ is a direct summand of $K$, that is, $K=B \oplus C$ for some $C \leq K$. If $C \neq 0$, then as at the beginning of the proof, we can find a pure subgroup of $X$ of $C$ of rank 1 . Clearly, $B \oplus X \in \Gamma$. Contradiction with maximality of $B$. So $C=0$. Then, $K$ is a direct summand of $\oplus_{i=1}^{\infty} G_{i}$. So $\oplus_{i=1}^{\infty} G_{i} \cong K \oplus \mathbb{Q}$. But $\oplus_{i=1}^{\infty} G_{i}$ is reduced. Contradiction. Thus, the process must be finite, that is, $N=G_{1} \oplus G_{2} \oplus \cdots \oplus G_{n}$ for some $n \in \mathbb{Z}^{+}$. To show that $N$ is pure-split, let $L$ be a pure subgroup of $N$. Then $M$ is $L$-pure-injective, so it is the direct sum of groups of rank one of finite number as we have proved above. Then, $L$ is a direct summand of $N$, because $N$-pure-injectiveness of $M$ implies that the inclusion $L \rightarrow N$ is splitting. Hence, $N$ is pure-split and completely decomposable with finite rank. This completes the proof.

Lemma 4.7. Let $N$ be a torsion-free group. If $M$ is an $N$-pure-injective, then $N$ is pure-split.

Proof. Let $K$ be a pure subgroup of $N=A \oplus D$, where $D$ is the divisible subgroup of $N$. Let $D_{K}$ be the divisible subgroup of $K$. Then $D_{K} \leq D$, and so $D=D_{1} \oplus D_{K}$ for some $D_{1} \leq D$. So $N=A \oplus D_{1} \oplus D_{K}=$ $E \oplus D_{K}$, where $E=A \oplus D_{1}$. By modular law, $K=(E \cap K) \oplus D_{K}$. Denote $E \cap K=L$. Then, $L \cong K / D_{K}$ is reduced and pure in $N$. Hence, $M$ is an $L$-pure-injective, and so $L \cong \bigoplus_{i=1}^{n} R_{i}$ for some rational groups $R_{1}, \ldots R_{n}$, by Lemma 4.6. Then, $M$ contains a direct summand isomorphic to $L$. So the inclusion $L \rightarrow N$ splits, i.e., $N=L \oplus H$ for some $H \leq N$. Since $L$ is reduced, $D_{K} \leq H$. Then $N=L \oplus D_{K} \oplus H^{\prime}=K \oplus H^{\prime}$. This implies that $N$ is pure-split.

Definition 4.8 (See, [6]). Let $G$ be a torsion-free group and $a \in G$. Given a prime $p$, the largest integer $k$ such that $p^{k} \mid a$ holds is called the $p$-height $h_{p}(a)$ of $a$; if no such maximal integer $k$ exists, then we set $h_{p}(a)=\infty$. The sequence of $p$-heights

$$
\chi(a)=\left(h_{p_{1}}(a), h_{p_{2}}(a), \ldots, h_{p_{n}}(a), \ldots\right)
$$

is said to be the characteristic of $a$. Two characteristics $\left(k_{1}, k_{2}, \ldots\right)$ and $\left(l_{1}, l_{2}, \ldots\right)$ are equivalent if $k_{n} \neq l_{n}$ holds only for a finite number of $n$ such that in case $k_{n} \neq l_{n}$ both $k_{n}$ and $l_{n}$ are finite. An equivalence class of characteristics is called a type. $G$ is called homogeneous if all nonzero elements of $G$ are of the same type.

Corollary 4.9. Let $N$ be a torsion-free reduced group. The following are equivalent.

(1) $M$ is an N-pure-injective.

(2) $N$ is pure-split.

(3) $N$ is a completely decomposable homogeneous group of finite rank.

Proof.

(1) $\Leftrightarrow(2) \quad$ By Lemma 4.6.

(2) $\Leftrightarrow(3) \quad$ See [4] or [6, Example 8, page 116].

Now, we can prove our theorem.

Proof of Theorem 4.1. Let $M$ be $G$-pure-injective for some group $G$. We have $G=N \oplus D$ for some reduced group $N$ and a divisible group $D$. Then $M$ is an $N$-pure-injective, and since $T(N)$ is a pure subgroup of $N, M$ is $T(N)$-pure-injective and $M$ is an $N / T(N)$-pure-injective. Then, by Lemmas 4.3 and 4.6, $T(N)=\oplus_{p \in P} B_{p}(N)$ and $N / T(N)=\oplus_{i \in I} K_{i}$, where for each $p \in P, B_{p}(N)$ is a bounded 
p-group, $I$ is a finite index set, and each $K_{i}$ is isomorphic to a rational group. We claim that $T(N)$ is a direct summand in $N$, that is, the short exact sequence:

$$
\mathbb{E}: 0 \rightarrow T(N) \rightarrow N \rightarrow N / T(N) \rightarrow 0
$$

is splitting. By [5, Theorem 52.2], there is a natural isomorphism

$$
\operatorname{Ext}(N / T(N), T(N))=\operatorname{Ext}\left(\bigoplus_{i \in I} K_{i}, T(N)\right) \cong \prod_{i \in I} \operatorname{Ext}\left(K_{i}, T(N)\right)
$$

induced by the inclusions $\alpha_{j}: K_{j} \rightarrow \bigoplus_{i \in I} K_{i}$. Therefore, it is sufficient to prove that each short exact sequence:

$$
\mathbb{E} \alpha_{j}: 0 \rightarrow T(N) \rightarrow N^{\prime} \stackrel{f}{\rightarrow} K_{j} \rightarrow 0
$$

is splitting. We have the following commutative diagram with exact columns and rows.

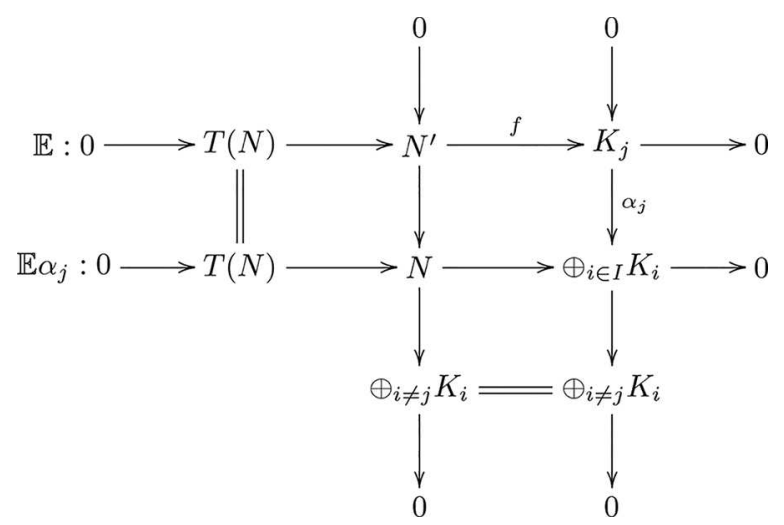

Since $\oplus_{i \in I} K_{i}$ is torsion free, $N^{\prime}$ is a pure subgroup of $N$, therefore $M$ is an $N^{\prime}$-pure-injective. There is a countable set $\left\{n_{k} \mid k=1,2, \ldots\right\}$ in $N^{\prime}$ such that the elements $f\left(n_{k}\right)$ generate $K_{j}$. By [5, Proposition 26.2], there is a countable pure subgroup $L$ of $N^{\prime}$ containing the subgroup $\sum_{k=1}^{\infty} \mathbb{Z} n_{k}$. Then, $M$ is an $L$-pureinjective as well. Clearly, $f(L)=K_{j}$ and $\operatorname{Ker}\left(\left.f\right|_{L}\right)=T(L)$. Since $L$ is countable, $T(L)$ is a countable subgroup of $T(N)$. But $T(N)$ is a direct sum of cyclic primary groups, therefore $T(L)$ is a countable direct sum of cyclic primary groups and hence is isomorphic to a direct summand of $M$. Since $T(L)$ is a subgroup of $L$ and $M$ is an $L$-pure-injective, $T(L)$ is a direct summand of $L$. We have the following commutative diagram with exact rows:

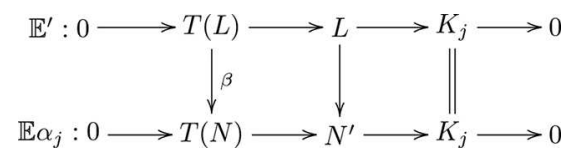

where $\beta$ is the inclusion. Since $\mathbb{E}^{\prime}$ is splitting $\mathbb{E} \alpha_{j}=\beta \mathbb{E}$ is also splitting. So $N=T(N) \oplus K$, where $T(N)$ and $K$ are groups as in Lemmas 4.3 and 4.6, respectively. This proves our claim.

To prove that $G$ is pure-split, take a pure subgroup $A$ of $G$. By the first part of the proof, we have

$$
G=N \oplus D=T(N) \oplus K \oplus T(D) \oplus D^{\prime}=(T(N) \oplus T(D)) \oplus\left(K \oplus D^{\prime}\right)=T(G) \oplus G^{\prime} .
$$

Then for each $p \in P, T_{p}(A)$ is a pure subgroup of $T_{p}(G)$. Therefore, $T_{p}(A)$ is a direct summand of $T_{p}(G)$ by Lemma 4.5. Then, $T(A)$ is a direct summand of $T(G)$. We have a homomorphism $f: A / T(A) \rightarrow$ $G / T(G)$ defined by $f(a+T(A))=a+T(G)$. If $f(a+T(A))=0$, then $a \in T(G) \cap A=T(A)$, hence $a+T(A)=0$, so $f$ is a monomorphism. Now claim that $\operatorname{Im}(f)$ is a pure subgroup of $G / T(G)$. To show this, let $a+T(G)=m(b+T(G))$ for some $a \in A, b \in G, 0 \neq m \in \mathbb{Z}$. Then $a-m b \in T(G)$, 
therefore $k a=k m b$ for some $0 \neq k \in \mathbb{Z}$. Since $A$ is pure in $G, k a=k m a^{\prime}$ for some $a^{\prime} \in A$. Then $a-m a^{\prime} \in T(A)$, hence $a+T(A)=m\left(a^{\prime}+T(A)\right)$. So $\operatorname{Im}(f)$ is pure. Since $G / T(G) \cong G^{\prime}$ is puresplit by Lemma 4.7, $f$ is splitting. As $A$ is a pure subgroup of $G, M$ is $A$-pure-injective. So again by the first part of the proof $A=T(A) \oplus K^{\prime}$ for some $K^{\prime} \leq A$ with $K^{\prime} \cong A / T(A)$. Then the inclusion map $A=T(A) \oplus K^{\prime} \rightarrow G=T(G) \oplus G^{\prime}$ is splitting, that is, $A$ is a direct summand in $G$. This completes the proof.

\section{Structure of pi-poor Abelian groups}

In this section, we prove some results concerning a possible structure of pi-poor groups.

Proposition 5.1. If $G$ is pi-poor group, then $T_{p}(G)$ is unbounded for each $p \in P$.

Proof. Suppose $G$ is pi-poor and $T_{p}(G)$ is bounded for some $p \in P$. Then $T_{p}(G)$ is pure-injective and $T_{p}(G)$ is a direct summand of $G$, because $T_{p}(G)$ is also pure in $G$. Consider the group $\oplus_{n=1}^{\infty} \mathbb{Z}_{p^{n}}$. We

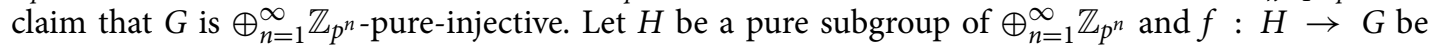
a homomorphism. Since $H$ is a $p$-group, $f(H) \subseteq T_{p}(G)$. So that $f$ extends to a homomorphism $h$ : $\oplus_{n=1}^{\infty} \mathbb{Z}_{p^{n}} \rightarrow G$ because $T_{p}(G)$ is pure-injective. This proves our claim.

We shall see that $\oplus_{n=1}^{\infty} \mathbb{Z}_{p^{n}}$ is not pure-split. There is an exact sequence:

$$
\mathbb{E}: 0 \rightarrow K \rightarrow \oplus_{n=1}^{\infty} \mathbb{Z}_{p^{n}} \stackrel{g}{\rightarrow} \mathbb{Z}_{p^{\infty}} \rightarrow 0 .
$$

By the same arguments as in the proof of Lemma $4.3, \mathbb{E}$ is pure, i.e., $K$ is pure in $\oplus_{n=1}^{\infty} \mathbb{Z}_{p^{n}}$. Since $\oplus_{n=1}^{\infty} \mathbb{Z}_{p^{n}}$ is reduced, $\mathbb{E}$ does not split. Hence $\oplus_{n=1}^{\infty} \mathbb{Z}_{p^{n}}$ is not pure-split. This contradicts with the fact that $G$ is pi-poor. Therefore, $T_{p}(G)$ can not be bounded.

Let $\mathbb{Q}_{p}$ be the localization of $\mathbb{Z}$ at the prime ideal $p \mathbb{Z}$. Note that the elements of $\mathbb{Q}_{p}$ are of the form $a b^{-1}$, where $a, b \in \mathbb{Z}, b \neq 0$, and $\operatorname{gcd}(b, p)=1$

Lemma 5.2. Let $p$ be a prime integer and $N$ be a reduced torsion group. Then for every homomorphism $f: \mathbb{Q}_{p} \rightarrow N, \operatorname{Im} f$ is bounded.

Proof. For every prime $q \neq p$, it is clear that $q \mathbb{Q}_{p}=\mathbb{Q}_{p}$, i.e., $\mathbb{Q}_{p}$ is $q$-divisible, and $T_{q}(N)$ is reduced. Then for $\pi_{q} \circ f: \mathbb{Q}_{p} \rightarrow T_{q}(N)$, where $\pi_{q}: N \rightarrow T_{q}(N)$ is the natural projection, $\left(\pi_{q} \circ f\right)\left(\mathbb{Q}_{p}\right)$ is a $q$-divisible subgroup of $T_{q}(N)$. Therefore, $\left(\pi_{q} \circ f\right)\left(\mathbb{Q}_{p}\right)$ is divisible, and so $\pi_{q} \circ f=0$ because $T_{q}(N)$ is reduced. Thus $\operatorname{Im} f=f\left(\mathbb{Q}_{p}\right) \subseteq T_{p}(N)$. Put $a=f(1)$ and $o(a)=p^{n}$, where $o(a)$ the order of $a$. Let $b c^{-1}$ be any element of $\mathbb{Q}_{p}$ with $\operatorname{gcd}(c, p)=1$. Then $\operatorname{gcd}\left(c, p^{n}\right)=1$, therefore $c y+p^{n} z=1$ for some $y, z \in \mathbb{Z}$. Now $b=b c y+b p^{n} z$, so $b c^{-1}=b y+b p^{n} z c^{-1}$. Note that $c f\left(b p^{n} z c^{-1}\right)=b z p^{n} f(1)=z p^{n} a=0$. Let $x=f\left(b p^{n} z c^{-1}\right)$ and $o(x)=p^{m}$. Since $\operatorname{gcd}\left(c, p^{m}\right)=1$, we have $c u+p^{m} v=1$ for some $u, v \in \mathbb{Z}$. Then $x=u c x+v p^{m} x=u c x=0$, and so $f\left(b c^{-1}\right)=f(b y)+x=f(b y)=b y f(1) \in\langle f(1)\rangle$. Hence $\operatorname{Im} f$ is contained in $\langle f(1)\rangle$, and so it is bounded.

A cotorsion group $G$ is a group satisfying $\operatorname{Ext}(\mathbb{Q}, G)=0$.

Theorem 5.3. There is a group $G$ such that $G$ is not pure-split and every reduced torsion group $N$ is G-pure-injective. Hence a pi-poor group can not be torsion.

Proof. Fix any prime $p$. Since $\mathbb{Q}_{p}$ is not cotorsion, $\operatorname{Ext}\left(\mathbb{Q}_{\mathbb{Q}} \mathbb{Q}_{p}\right) \neq 0$ (see [5], page 226, Example 15). So there is a nonsplitting pure sequence:

$$
0 \rightarrow \mathbb{Q}_{p} \rightarrow G \rightarrow \mathbb{Q} \rightarrow 0
$$


Hence, $G$ is not pure-split. For every prime $q \neq p, \mathbb{Q}_{p}$ and $\mathbb{Q}$ are $q$-divisible, therefore $G$ is also $q$-divisible. We claim that $N$ is $G$-pure injective. Without loss of generality, we can assume that $\mathbb{Q}_{p}$ is a subgroup of $G$ and $G / \mathbb{Q}_{p}=\mathbb{Q}$. Let $K$ be any nonzero pure subgroup of $G$ and $f: K \rightarrow N$ be any homomorphism, where $N$ is a torsion reduced group. Then, $K$ is $q$-divisible for every prime $q \neq p$ since $K$ is a pure subgroup of $G$ and $G$ is $q$-divisible. Clearly, the rank of $K$ is at most 2. So have two cases:

Case I: $\quad r_{0}(K)=1$. If $K$ is also $p$-divisible, then $K$ is divisible. So $K \cong \mathbb{Q}$, and the inclusion $K \rightarrow G$ splits, so $f$ can be extended to a homomorphism $f^{\prime}: G \rightarrow N$. Now, let $K$ be not $p$-divisible. $K$ and $\mathbb{Q}_{p}$ are of the same type, and so $K \cong \mathbb{Q}_{p}$ (see [5, Theorem 85.1]). Therefore, $\operatorname{Im} f$ is bounded by Lemma 5.2. Then, $\operatorname{Im} f$ is pure-injective, hence $f: K \rightarrow N$ can be extended to a homomorphism $f^{\prime}: G \rightarrow \operatorname{Im} f \leq N$.

Case II: $\quad r_{0}(K)=2$ : We claim that $K=G$. Otherwise, since $G / K$ is a nonzero torsion-free group, $r_{0}(G / K) \geq 1$. Then $2=r_{0}(G)=r_{0}(K)+r_{0}(G / K)>2$, a contradiction. Hence $G=K$.

As a consequence, $N$ is $G$-pure-injective. This implies that $N$ is not pi-poor.

Corollary 5.4. Let $M$ be a pi-poor group. Then $M \neq T(M)$ and $T_{p}(M)$ is unbounded for every $p \in P$.

Lemma 5.5. Let $M$ and $N$ be right $R$-modules. Assume that $N$ is (pure-)injective. Then, $M \oplus N$ is (pi-)poor if and only if $M$ is (pi-)poor.

Proof. For a right $R$-module $B$, it is clear that $M \oplus N$ is $B$-(pure-)injective if and only if $M$ is $B$ (pure-)injective.

Example 5.6. Let $G=\oplus_{p \in P} \mathbb{Z}_{p}$. Then $G$ is poor by Theorem 3.1. On the other hand, since $T_{p}(G)=\mathbb{Z}_{p}$ is bounded, $G$ is not pi-poor by Proposition 5.1.

Example 5.7. Let $M$ be as in Theorem 4.1 and let $V$ be the sum of all direct summands isomorphic to $\mathbb{Z}_{p}$. If $M=V \oplus K$, then $K$ is pi-poor by Lemma 5.5. But $K$ is not poor by Theorem 3.1, since $K$ does not contain a direct summand isomorphic to $\mathbb{Z}_{p}$. So pi-poor modules need not be poor.

\section{Acknowledgment}

The authors are grateful to the referee for the suggestions and comments which improved the presentation of the paper.

\section{References}

[1] Alahmadi, A. N., Alkan, M., López-Permouth, S. (2010). Poor modules: The opposite of injectivity. Glasg. Math. J. 52(A):7-17.

[2] Anderson, F. W., Fuller, K. R. (1992). Rings and Categories of Modules. New York: Springer.

[3] Er, N., López-Permouth, S., Sökmez, N. (2011). Rings whose modules have maximal or minimal injectivity domains. J. Algebra 330:404-417.

[4] Fuchs, L., Kertész, A., Szele, T. (1953). Abelian groups in which every serving subgroup is a direct summand. Publ. Math. Debrecen 3:95-105 (1954).

[5] Fuchs, L. (1970). Infinite Abelian Groups. Vol. I. Pure and Applied Mathematics, Vol. 36. New York: Academic Press.

[6] Fuchs, L. (1973). Infinite Abelian Groups. Vol. II. Pure and Applied Mathematics. Vol. 36-II. New York: Academic Press.

[7] Harmanc1, A., López-Permouth, S., Üngör, B. (2015). On the pure-injectivity profile of a ring. Commun. Algebra, 43-11:4984-5002.

[8] Mohamed, S. H., Müller, B. J. (1990). Continuous and Discrete Modules. London Mathematical Society Lecture Note Series, Vol. 147. Cambridge: Cambridge University Press. 